\title{
DNA methyltransferase inhibitors influence on the DIRAS3 and STAT3 expression and in vitro migration of ovarian and breast cancer cells
}

\author{
Ewa Maria Nowak, Marta Poczęta, Dominik Bieg, Ilona Bednarek \\ Department of Biotechnology and Genetic Engineering, School of Pharmacy with the Division of Laboratory Medicine \\ in Sosnowiec, Medical University of Silesia in Katowice, Poland
}

\begin{abstract}
Objectives: Downregulation of DIRAS3 (DIRAS family, GTP-binding Ras-like 3) is related to ovarian and breast cancer progression. A possible mechanism that silences this gene is the promoter region DNA methylation. The potential reversibility of this epigenetic mechanism makes it more attractive candidate for new mode of cancer treatment. DIRAS3 regulates cell cycle, tumor dormancy and inhibits cancer cell growth and motility, all of which may indirectly depend on interaction with STAT3 (Signal Transducer and Activator of Transcription 3) classified as a potential oncogene. The restoration of DIRAS3 expression could inhibit cell proliferation and invasiveness.
\end{abstract}

Material and methods: Human ovarian carcinoma cell line (A2780) and human breast cancer cell line (MCF7) were exposed to two DNA methyltransferase inhibitors (DNMTi): decitabine (5-aza-2'-deoxycytidine) $[25 \mu \mathrm{M}$ and $12.5 \mu \mathrm{M}]$ and RG108 [150 $\mu \mathrm{M}$ and $100 \mu \mathrm{M}]$. In vitro migration changes of cancer cells were examined with wound healing assay. After 7 days of DNMTi treatment cells were harvested and DNA and RNA was isolated. The methylation status of the promoter sequences of DIRAS3 and STAT3 genes was determined using methylation specific PCR (MS-PCR). Level of target genes' expression was quantified using quantitative reverse transcription PCR (QRT-PCR).

Results and conclusions: The in vitro wound healing assay showed changes in the migration rate of both adherent cell lines after DNMTi treatment compared to the untreated cells. Relative balance between methylated and unmethylated variants of DIRAS3 after MS-PCR was shifted towards unmethylated version after DNMTi treatment in A2780 cells. Statistically significant dose dependent effect of decitabine and RG108 on DIRAS3 expression in A2780 cells was observed.

Key words: DNA methylation, DNA methyltransferases inhibitors, DIRAS3 expression, STAT3 expression, ovarian cancer, breast cancer

Ginekologia Polska 2017; 88, 10: 543-551

\section{INTRODUCTION}

Epigenetic changes in ovarian and breast cancer

Cancer initiation and progression is caused by various genetic abnormalities, such as somatic mutations or genomic instability, which leads to activation of oncogenes and inactivation of tumor suppressor genes. Furthermore, DNA methylation and post-translational histone modifications being well-known epigenetic changes also play an important role in tumorigenesis [1]. DNA methylation is catalyzed by methyltransferases (DNMT1, DNMT3A, DNMT3B, and DNMT3L) which are responsible for the addition of a methyl group to the carbon-5 position of the cytosine pyrimidine ring in the $5^{\prime}-\mathrm{CpG}-3^{\prime}$ dinucleotide. DNMT1 is involved in the maintenance of methylation ensuring merely the methylation pattern fidelity of replicated daughter strands, whereas DNMT3 is a de novo methyltransferase [2].

The two commonly described methylation patterns in ovarian or breast cancer are hypermethylation of promoters, which most likely leads to silencing of gene and thus facilitating cancer formation, and hypomethylation of highly repeated DNA sequences, which potentially leads to aberrant expression of oncogenic genes [3, 4]. The presence of hypermethylated promoter affects genes involved in cell cycle, cell adhesion, apoptosis or DNA repair. It may cause 
the inactivation of certain tumor suppressor genes, which is observed in different cancer types [5].

\section{DIRAS3 and STAT3 - possible mechanisms of regulation of gene expression}

DIRAS3 (DIRAS family, GTP-binding Ras-like 3) also known as ARHI (aplysia ras homolog I) or NOEY2 is a protein coding gene located on chromosome 1p31.3. The gene contains two exons interrupted by a large intron. About $1 \mathrm{~kb}$ upstream of the transcription initiation site the first CpG island is found. The second island is found near the transcription region and the third $\mathrm{CpG}$ island is found in the exon 2 where a protein-coding region is located [6]. DIRAS3 is a maternally imprinted tumor suppressor gene susceptible to loss of paternal allele expression in the process of $\mathrm{LOH}$ (loss of heterozygosity) or by epigenetic modifications like, transcriptional regulation or changes in methylation pattern. When expressed, this gene encodes a 26-kDa GTPase that belongs to the RAS superfamily of small $G$ proteins with high homology to RAS and RAP but with a distinctive 34 amino acid N-terminal extension [7]. It is expressed inter alia in the ovary and breast epithelial cells. A loss or downregulation of its expression is associated with the majority of ovarian and breast cancers [8-10]. DIRAS3 regulates cell cycle, autophagy, motility, tumor dormancy and inhibits cancer cell growth, which may indirectly depend on its interaction with STAT3 (Signal Transducer and Activator of Transcription 3) that has been classified as a potential oncogene. A phosphorylated and activated STAT3 translocates to the nucleus where it increases transcription of other oncogenes in the tumor cells. On the other hand, unphosphorylated STAT3 may also activate gene expression by an IL-6-dependent activation [11]. Overexpression of this gene has been observed in breast and ovarian cancer [11-13]. STAT3 translocation to nucleus can be inhibited by DIRAS3 via direct interaction or by down regulation of $\beta$-integrin expression. However, it has been shown that acetylated STAT3 is involved in regulating the methylation pattern of some tumor suppressor genes e.g., it stimulates the methylation of the DIRAS3 promoter region, which results in down regulation of transcription [12].

\section{DNMTi - mechanism of action of nucleoside and non-nucleoside analogues}

There are different well-characterized strategies leading to inhibition of DNA methylation including DNMTs antisense oligonucleosides or DNA methyltransferase inhibitors (DNMTi) being either nucleoside analogues or non-nucleoside compounds [1]. Nucleoside analogues like azacitidine (5-azacitidine), decitabine (5-aza-2'-deoxycytidine), zebularine (1-[(2R,3R,4S,5R)-3,4-dihydroxy-5-(hydroxymethyl) oxolan-2-yl]pyrimidin-2-one), DHAC (5,6-dihydro-5-aza- cytidine) and FCDR (5-fluoro-2'-deoxycytidine) are called first generation compounds. Some of them have already been approved (azacitidine [Vidaza ${ }^{\circledR}$, Celgene] in 2004 and decitabine [Dacogen ${ }^{\oplus}$, Janssen-Cilag] in 2006) by the FDA (Food and Drug Administration) [14] for treating blood-related diseases like myelodysplastic syndrome (MDS) and acute myeloid leukemia (AML). Yet, their activity against solid tumors is still under investigation (phase II of clinical research) [15]. Nucleoside-like inhibitors (the analogues) are first transported into cells by human concentrative nucleoside transporter (hCNT1) and then converted into active triphosphate forms that are integrated into the genome during $S$ phase of cell cycle. Azacitidine, since it is a ribose analogue, is incorporated into RNA and after deoxy-conversion into DNA. Decitabine as a deoxyribose analogue is incorporated only into DNA by DNA polymerase and for this reason it is more active than azacitidine $[15,16]$. The incorporated inhibitors trap the arriving enzyme by irreversible covalent binding with the catalytic cysteine of DNMTs, which prevents DNA synthesis and subsequently leads to toxicity, even if such trapped enzyme promotes its proteosomal degradation $[17,18]$.

In turn, non-nucleoside DNMT inhibitors directly block the enzyme's catalytic site rendering it non-functional so its associated toxicity is lower. A three-dimensional model of DNMT1 catalytic domain aids at the search for such direct inhibitors (like tryptophan derivatives - RG108). Some of such inhibitors are used in the treatment of disorders where their DNMTs inhibitory activity comes as a secondary therapeutic effect (for instance procainamide derivatives reduce the affinity of DNMT1 to DNA).

\section{OBJECTIVES}

The analysis of the aberrant DNA methylation patterns and the hypermethylated promoters can provide information about silenced tumor suppressor genes so to identify useful biomarkers of cancer progression. Moreover, for a reversible mechanism such as the hypermethylation of DNA promoters, the use of DNMT inhibitors represents a promising approach to cancer therapy. The main aim of this study is to investigate the potential influence of DNMTi on the expression of DIRAS3 and STAT3 genes in reference to changes in their promoters' methylation level and in reference to changes in migration of cancer cells.

\section{MATERIAL AND METHODS Cell culture}

Human ovarian carcinoma cell line (A2780, European Collection of Cell Culture, ECACC ${ }^{\circledR}$ 93112519) used in the study was established from tumor tissue of untreated patient. Human breast cancer cell line (MCF7, American Type 
Culture Collection, ATCC ${ }^{\circledR}$ HTB- $22^{\text {TM}}$ ) used in the study was derived from pleural effusion as a metastatic site.

Cells were cultured in RPMI-1640 medium (Biowest) including L-glutamine, supplemented with $10 \%(\mathrm{v} / \mathrm{v})$ fetal calf serum (Biological Industries) and $0.1 \%(\mathrm{v} / \mathrm{v})$ gentamicin $(50 \mathrm{mg} / \mathrm{mL}$ gentamicin sulfate, Biological Industries) in a humidified atmosphere containing $5 \% \mathrm{CO}_{2}$ at $37^{\circ} \mathrm{C}$.

\section{Treatment with DNMTi}

Decitabine (5-aza-2'-deoxycytidine, Sigma-Aldrich) and RG108 (N-Phthalyl-L-tryptophan, Sigma-Aldrich) 10 mM stock solutions were prepared in a DMSO (dimethyl sulfoxide) and stored at $-20^{\circ} \mathrm{C}$. Cells were exposed to different concentrations of DNMTi (5-aza-2'-deoxycytidine of $25 \mu \mathrm{M}$ and $12.5 \mu \mathrm{M}, \mathrm{RG} 108$ of $150 \mu \mathrm{M}$ and $100 \mu \mathrm{M}$ ) that were selected after a prior cytotoxicity test evaluation (data not shown). The medium supplemented with inhibitors was changed every day until cell harvesting 7 days later. Untreated control cells were cultured in a cell growth medium concurrently.

\section{Wound healing assay}

Migration and motility changes of cancer cells were examined with a wound healing assay. Similarly, cells treated with DNA methyltransferase inhibitors and control cells were seeded to achieve approximately $60-80 \%$ confluence. Using a sterile pipette tip (volume $20-200 \mu \mathrm{L}$ ) a straight scratch in the cell's monolayer was made to simulate a wound. After scratching the medium was changed for a fresh growth medium supplemented with decitabine and RG108. The monolayers were then incubated at $37^{\circ} \mathrm{C}$ for 7 days. The in vitro healing and the speed of cells' movement across the gap were observed. Digital documentation was made after scratching (at time zero) and after 7 days by an inverted microscope (Axiovert, Zeiss). The impact of applied modulators on the migration of cells was estimated with a relative distance of wound closure.

\section{RNA isolation and QRT-PCR}

Total RNA was prepared using the $Z$ R-Duet ${ }^{\mathrm{TM}}$ DNA/RNA MiniPrep (Zymo Research) according to the manufacturer's protocol. Total RNA was converted to CDNA and relative quantitation of gene expression was performed with Brilliant II SYBR ${ }^{\circledast}$ Green QRT-PCR Master Mix Kit, 1-Step (Agilent Technologies). A quantitative reverse transcription
PCR (QRT-PCR) was performed on STRATAGENE Mx3000P to investigate the expression of DIRAS3, STAT3 and TBP (the housekeeping gene encoding TATA-box binding protein). A similar thermal profile was used for all the reactions: initial cDNA synthesis step of $30[\mathrm{~min}]$ at $50^{\circ} \mathrm{C}$, then 15 [min] at $95^{\circ} \mathrm{C}$ followed by 40 cycles of $15[\mathrm{~s}]$ at $94^{\circ} \mathrm{C}, 60[\mathrm{~s}]$ at $65^{\circ} \mathrm{C}$ (for DIRAS3) or 60 [s] at $60^{\circ} \mathrm{C}$ (for STAT3 and TBP), and 30 [s] at $72^{\circ} \mathrm{C}$, followed by $10 \mathrm{~min}$ at $72^{\circ} \mathrm{C}$.

All the primers (sequences presented in Table 1) were synthesized by DNA Sequencing and Oligonucleotide Synthesis Laboratory of the Institute of Biochemistry and Biophysics at Polish Academy of Sciences in Warsaw.

The level of target genes expression was quantified using relative quantification. The $\Delta \mathrm{Cq}$ value for each sample was determined by calculating the difference between the $\mathrm{Cq}$ (quantitation cycle) value of the target genes (DIRAS3 and STAT3) and the Cq value of the endogenous reference gene (TBP). This was determined for every sample of cells treated with decitabine and RG108 and for the control untreated cells as calibrator sample. Next, the $\Delta \Delta C$ q value for each sample was determined by subtracting the $\Delta C q$ value of the calibrator from the $\Delta \mathrm{Cq}$ value of particular samples. Finally, the normalized level of target gene expression was calculated by using the formula: $2^{-\Delta \Delta C q}[19]$.

\section{DNA isolation and MS-PCR}

Total DNA was prepared using the ZR-Duet ${ }^{\mathrm{TM}}$ DNA/RNA MiniPrep (Zymo Research) according to the manufacturer's protocol. The methylation status of the promoter sequences of DIRAS3, STAT3 and TBP genes was determined using methylation specific PCR (MS-PCR). For every subsequent MS-PCR a bisulfite modification of the DNA sample was carried out. After bisulfite treatment with Methylation-Gold ${ }^{\mathrm{TM}}$ Kit (Zymo Research), unmethylated cytosines were converted to uracils, while 5-methylcytosines remained unaltered. Next, bisulfite modified DNA was amplified by using two different sets of primer pairs, of which one pair (M) recognized the methylated alleles while the another pair (uM) recognized the unmethylated alleles of the promoter region of genes. All the primers (sequences presented in Table 2) were designed with MethPrimer (http://www.urogene.org/methprimer/index.html) and synthesized by DNA Sequencing and Oligonucleotide Synthesis Laboratory of the Institute of Biochemistry and Biophysics at Polish Academy of Sciences in Warsaw.

Table 1. Primers sequences with the amplification products length

\begin{tabular}{|l|l|l|l|}
\hline Gene & Forward primer & Reverse primer & Product \\
\hline DIRAS3 & 5'TGCCTCCGAGAAAGGGGTCT3' $^{\prime}$ & 5'CCAAAGCTGGCGTTACCCAT3' & $99 \mathrm{bp}$ \\
\hline STAT3 & 5'GAAAACATGGCTGGCAAGGG3' & 5'GCCCATGATGTACCCTTCGT3' & $108 \mathrm{bp}$ \\
\hline TBP & 5'TATAATCCCAAGCGGTTTGCTG3' & 5'GCCAGTCTGGACTGTTCTTCA3' & $125 \mathrm{bp}$ \\
\hline
\end{tabular}


Table 2. Primers sequences with the amplification products length ( $M$ - methylated, uM - unmethylated)

\begin{tabular}{|c|c|c|c|}
\hline Gene & Forward primer & Reverse primer & Product \\
\hline DIRAS3 M & 5'TATAGGTAAGGGAGAAAGAAGTTAGAC3' & 5'GACTAAAAAACCCGATTATATCGTT3' & $130 \mathrm{bp}$ \\
\hline DIRAS3 uM & 5'TAGGTAAGGGAGAAAGAAGTTAGATG3' & 5'AACTAAAAAACCCAATTATATCATT3' & $128 \mathrm{bp}$ \\
\hline STAT3 M & 5'GGTTTCGGTTGTATCGTATACGT3' & 5'CCGATTAAAACTTATTCCCTCG3' & $218 \mathrm{bp}$ \\
\hline STAT3 UM & 5'GGGGTTTTGGTTGTATTGTATATGT3' & 5'AAATCCAATTAAAACTTATTCCCTCA3' & 224 bp \\
\hline TBPM & 5'TTCGGTTTTAGTGGGAGTAATTC3' & 5'GAAAACGAAATTAAACAACCGAC3' & $143 \mathrm{bp}$ \\
\hline TBP uM & 5'TTTTGGTTTTAGTGGGAGTAATTTG3' & 5'CAACAAAAACAAAATTAAACAACCA3' & $148 \mathrm{bp}$ \\
\hline
\end{tabular}

For all MS-PCR reactions a similar thermal profile was used: initial denaturation step of $10[\mathrm{~min}]$ at $95^{\circ} \mathrm{C}$ followed by 40 cycles of $30[\mathrm{~s}]$ at $95^{\circ} \mathrm{C}, 30[\mathrm{~s}]$ at $57^{\circ} \mathrm{C}$ (for DIRAS3 amplification) or $30[\mathrm{~s}]$ at $59^{\circ} \mathrm{C}$ (for STAT3 and TBP amplification), and $40[\mathrm{~s}]$ at $72^{\circ} \mathrm{C}$, followed with a final extension at $72^{\circ} \mathrm{C}$ for 7 [min].

The MS-PCR products were separated on $2 \%$ agarose gels (Agarde Ultra, Roth) and stained with ethidium bromide (final concentration: $0.5 \mu \mathrm{g} / \mathrm{mL}$ ) with DNA pUC Mix Marker 8 (concentration: $0.1 \mu \mathrm{g} / \mu \mathrm{L}$, Thermofisher). Electropherograms were visualized under UV illumination using GENIUS IN Syngene Bio Imaging (Syngene) gel documentation system. Next, images were analyzed using ImageJ software.

\section{Statistical analysis}

All results were analyzed statistically using the STATISTICA 12.5 software. The Shapiro-Wilk test was used to determine normality. The level of significance was set at $p<0.05$.

Genes expression levels were compared using Student's $t$ test while statistical comparison of migration rate change was performed using (ANOVA). Tukey's test was used for the analysis of significance between different values.

\section{RESULTS}

The effect of DNA methylation inhibitors on gene expression and DNA methylation

\section{Gene expression changes in A2780 and MCF-7 in response to treatment with decitabine and RG108}

DIRAS3 expression is reduced in ovarian and breast cancer cell lines, which has already been described in the literature. Several mechanisms may be responsible for such reduction of expression including primarily promoter hypermethylation. Consequently, this can be addressed with DNMT inhibitors as treatment of ovarian and breast carcinoma, which is a promising idea since epigenetic changes are reversible.

We observed some statistically significant dose dependent effects of decitabine and RG108 on DIRAS3 expression in A2780 cells. Compared to control cells decitabine has augmented the expression level of DIRAS3 mRNA 3.16-fold and 3.53-fold $(p<0.05)$ at the dose of $12.5 \mu \mathrm{M}$ and $25 \mu \mathrm{M}$ respectively. In turn, RG108 has augmented the expression of DIRAS3 mRNA 5.06-fold and 3.32-fold ( $p<0.05$ )

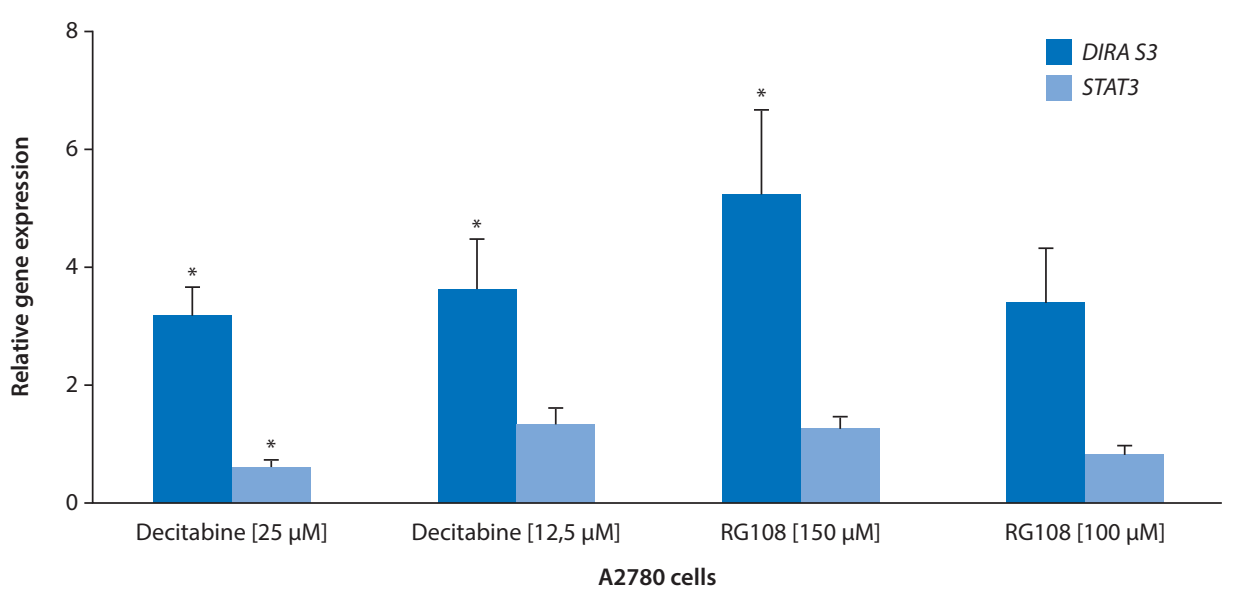

Figure 1. Comparison of normalized DIRAS3 and STAT3 expression level (calculated with the $2^{-\triangle \Delta C a}$ formula) in A2780 cells treated with decitabine [25 and $12.5 \mu \mathrm{M}]$ and RG108 [150 and $100 \mu \mathrm{M}]$ in reference to control cells; ${ }^{*}$ statistically significant results $(p<0.05)$ 
at respective dose of $150 \mu \mathrm{M}$ and $100 \mu \mathrm{M}$ (Fig. 1). On the other hand, decitabine and RG108 treatment of MCF7 cells showed no influence on the activation of DIRAS3 expression (Fig. 2).

Similarly, we have also observed statistically significant $(p<0.05)$ dose dependent effects of decitabine on STAT3 expression. Decitabine at the dose of $25 \mu \mathrm{M}$ has decreased the expression of STAT3 in A2780 cells compared to control cells (Fig. 1).

Relative quantitation of STAT3 expression showed no changes after exposure to $12.5 \mu \mathrm{M}$ decitabine and RG108 treatment compared to A2780 control cells.

Decitabine and RG108 treatment of MCF7 cells showed no influence on the activation of STAT3 expression.

\section{The DNA methylation status of promoters of targeted genes in A2780 and MCF-7 cells in response to treatment with decitabine and RG108}

Electrophoretic analysis of the MS-PCR results showed partial methylation of DIRAS3 gene promoter fragment in the A2780 control cells. Relative balance between methylated and unmethylated variants was shifted towards unmethylated genes after treatment with DNA methyltransferase inhibitors. Relative density of bands determined by ImageJ software showed a $37.5 \%$ and $41.6 \%$ decrease of methylation variants after decytabine and RG108 treatment respectively and a $136.5 \%$ and $59.3 \%$ increase of unmethylated variants after decytabine and RG108 treatment respectively, all compared to control cells. Electrophoretic analysis of the MS-PCR results of the STAT3 and TBP promoter fragments showed the dominance of unmethylated variant in control cells as well as in the cells treated with decitabine and RG108. Similar electrophoretic results of MS-PCR of DIRAS3, STAT3 and TBP fragments of genes' promoters were observed in MCF7 cells.
Relative density of bands determined by ImageJ software showed a $13.9 \%$ and $9.7 \%$ decrease of methylation variants after decytabine and RG108 treatment respectively compared to control cells. All results are presented in Figure 3. The influence of reducing the methylation level within the promoter sequence on the increasing DIRAS3 expression was confirmed in A2780 cells.

\section{Effect of DNA methylation inhibitors on the cells migration}

To evaluate the possible influence of DNMT inhibitors on the increase of DIRAS3 expression we have examined the cellular migration of A2780 and MCF7 cells using an in vitro wound healing assay. The observed changes in the migration rate of both adherent cell lines were compared to the control group of untreated cells. Representative images of in vitro wound healing assay are shown in the Figure 4 . We noticed a significant delay in wound closure in both experimental groups of cells treated with decitabine or RG108 compared to the control group. The rate of wound healing was defined as the average distance between the wound edges.

Decitabine treated $\mathrm{A} 2780$ cells have covered the wound in $12.85 \%$ [ $25 \mu \mathrm{M}$ ] and $14.87 \%$ [12.5 $\mu \mathrm{M}$ ], whereas A2780 cells treated with RG108 covered the wound in 5.77\% [150 $\mu \mathrm{M}]$ and $15.48 \%$ [100 $\mu \mathrm{M}]$. After 7 days, A2780 control cells covered the wound in $88.11 \%$ (Fig. 5). MCF7 cells treated with decitabine covered the wound in 44.97\% [25 $\mu \mathrm{M}]$ and $36.62 \%[12.5 \mu \mathrm{M}]$, whereas cells treated with RG108 covered the wound in $34.97 \%$ [150 $\mu \mathrm{M}$ ] and 47.85\% [100 $\mu \mathrm{M}$ ]. After 7 days, MCF7 control cells covered the wound in $85.53 \%$ (Fig. 6). To sum up, both DNMT inhibitors have significantly $(P<0.001)$ reduced the speed of in vitro A2780 cells migration in comparison to untreated control cells cultured in cell growth medium after 7 days. Changes in speed migration of

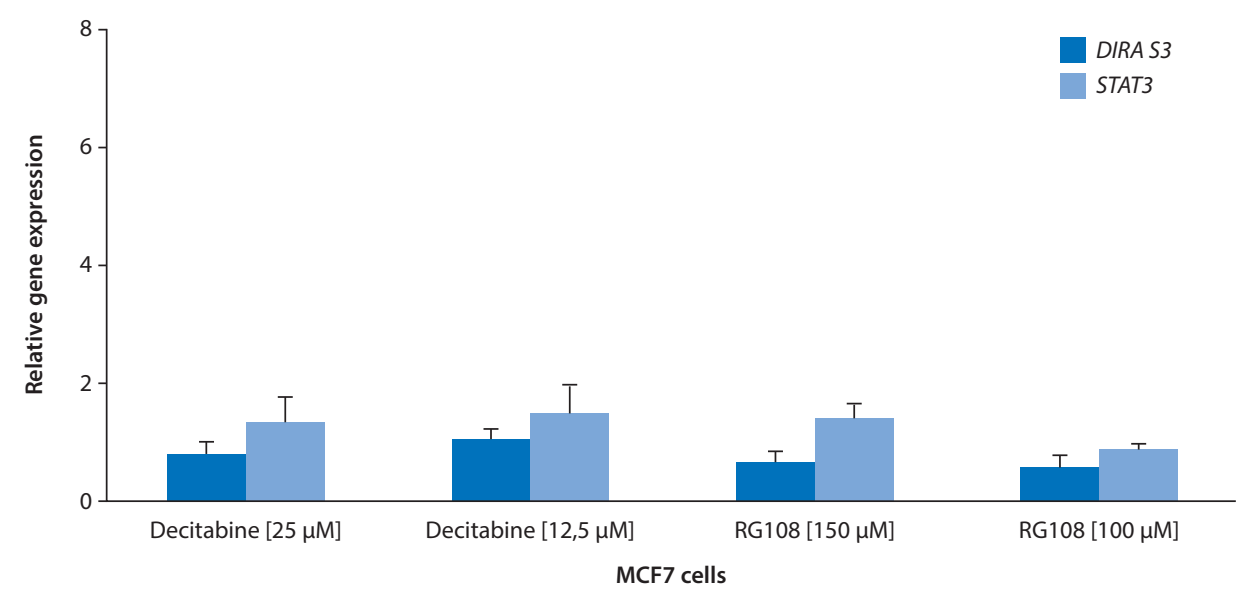

Figure 2. Comparison of normalized DIRAS3 and STAT3 expression level (calculated with the $2^{-\triangle \Delta C q}$ formula) in MCF7 cells treated with decitabine [25 and $12.5 \mu \mathrm{M}]$ and RG108 [150 and $100 \mu \mathrm{M}]$ in reference to control cells 


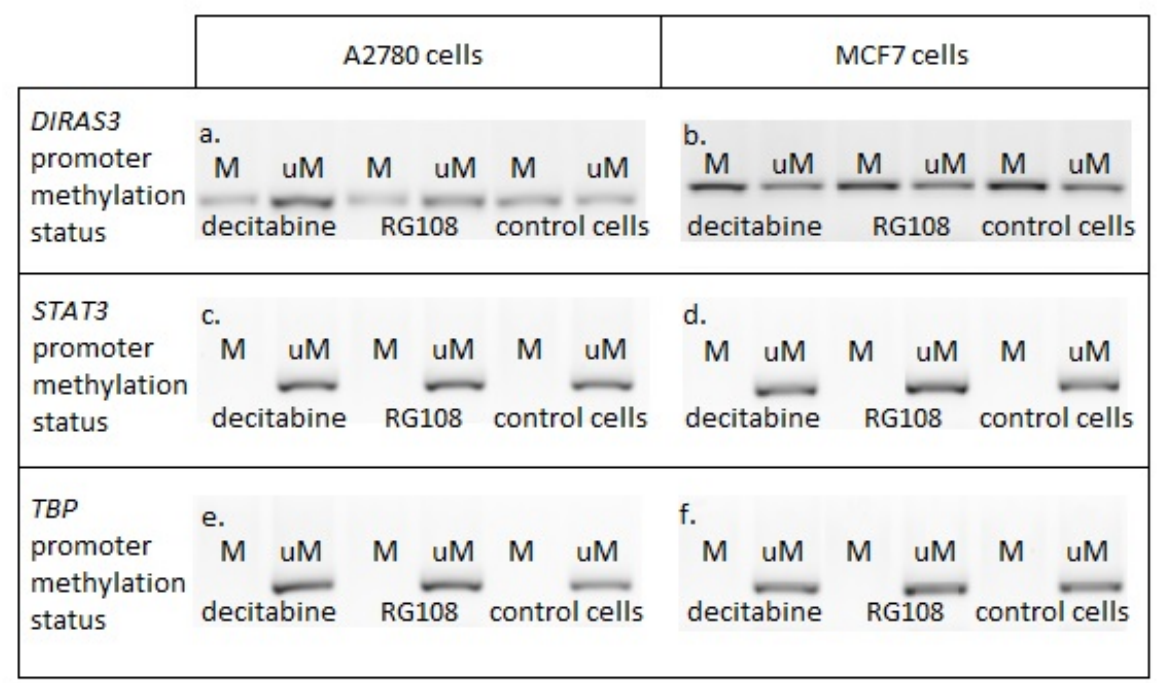

Figure 3. Methylation status of the fragment of promoter region of DIRAS3, STAT3 and TBP gene in A2780 (a., c., e.) cells treated with decitabine $[25 \mu \mathrm{M}]$ and RG108 $[150 \mu \mathrm{M}]$ in comparison to control cells. Methylation status of the fragment of promoter region of DIRAS3, STAT3 and TBP gene in MCF7 cells (b., d., f.) treated with decitabine $[25 \mu \mathrm{M}]$ and RG108 [150 $\mu \mathrm{M}]$ in comparison to control cells. Methylation status was examined by MS-PCR with a methylated primer set (M) and an unmethylated primer set (UM)

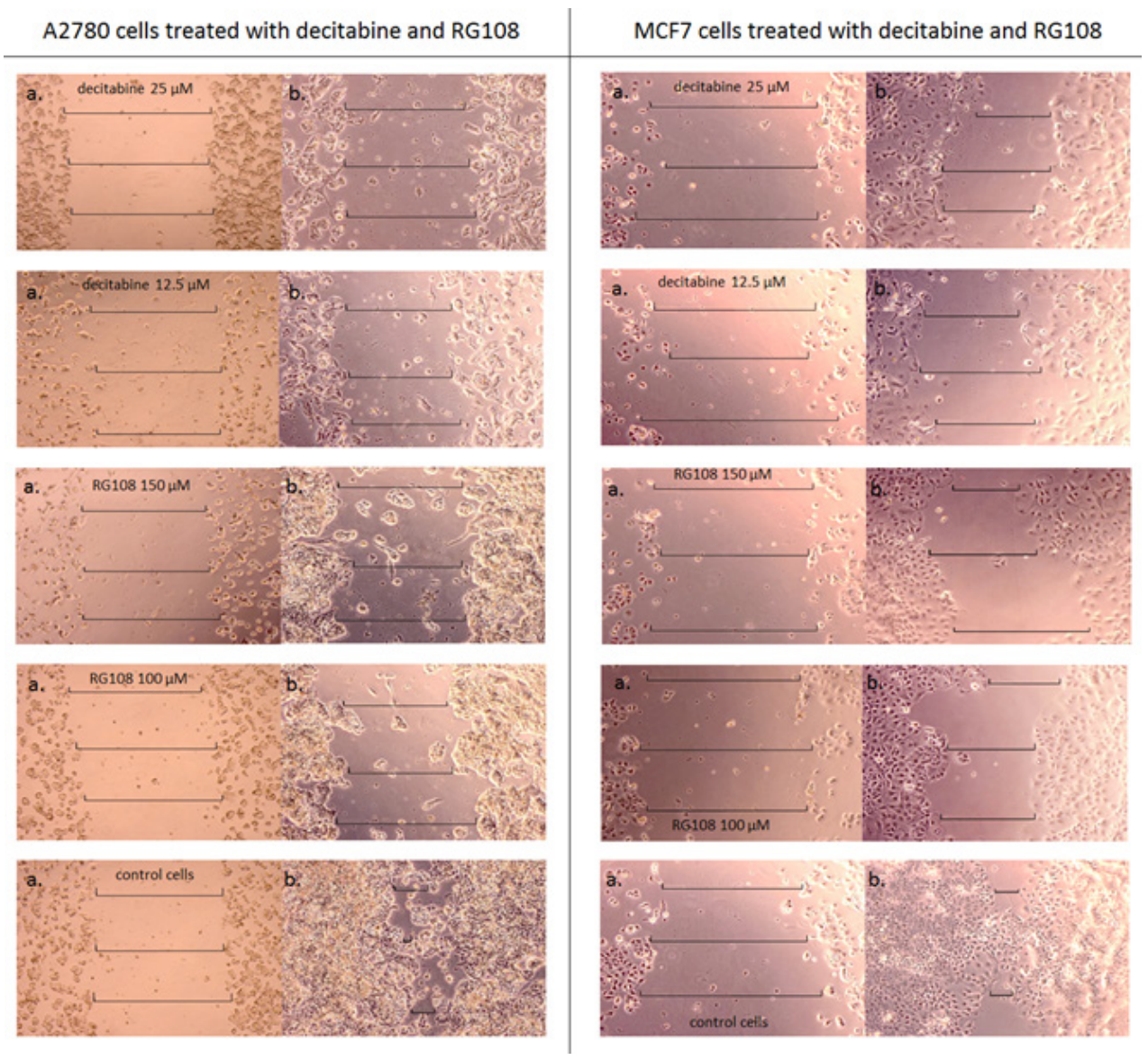

Figure 4. The effect of decitabine and RG108 on the cell migration and motility was estimated by the relative distance of wound closure compared to control cells. Digital documentation was made after scratching - at time zero (a.) and after 7 days (b.) by inverted microscope (Axiovert, Zeiss) 


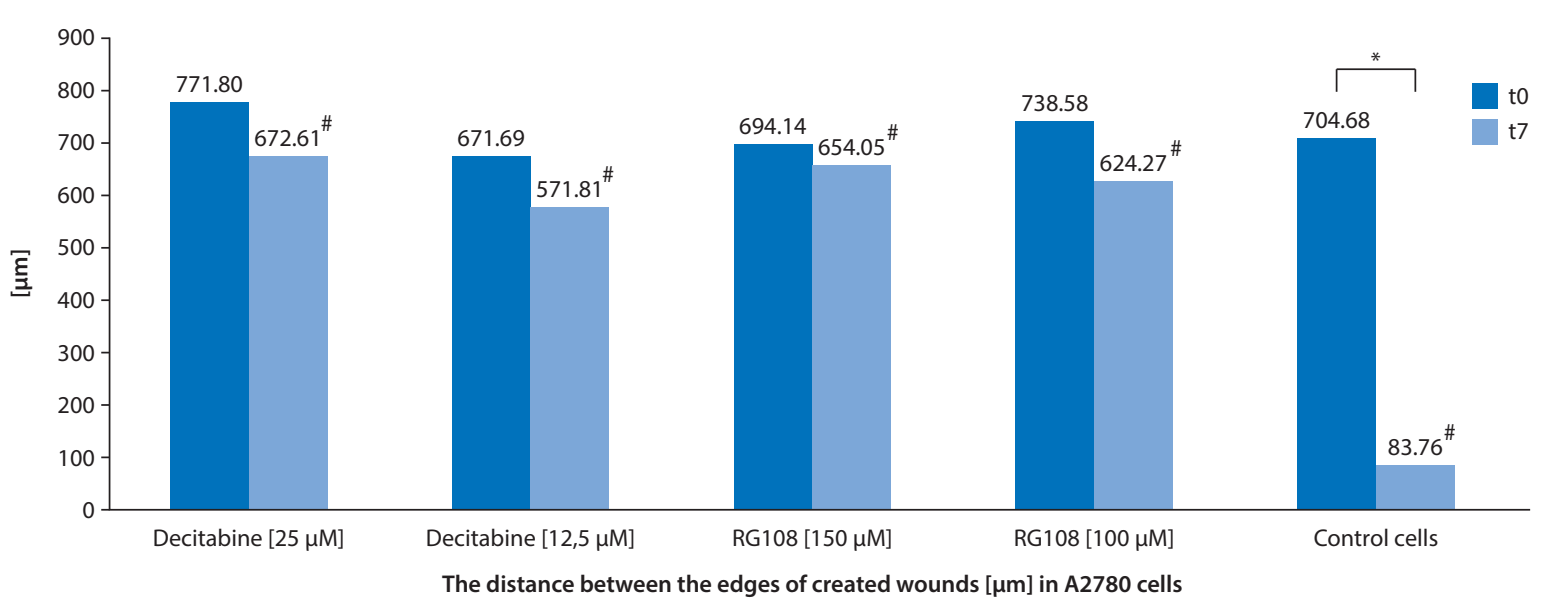

Figure 5. Changes in the average distance between the edges of created wounds in A2780 cells [ $\mu \mathrm{m}]$ treated with DNMTi and in control cells, *, \# statistically significant results $(p<0.001)$

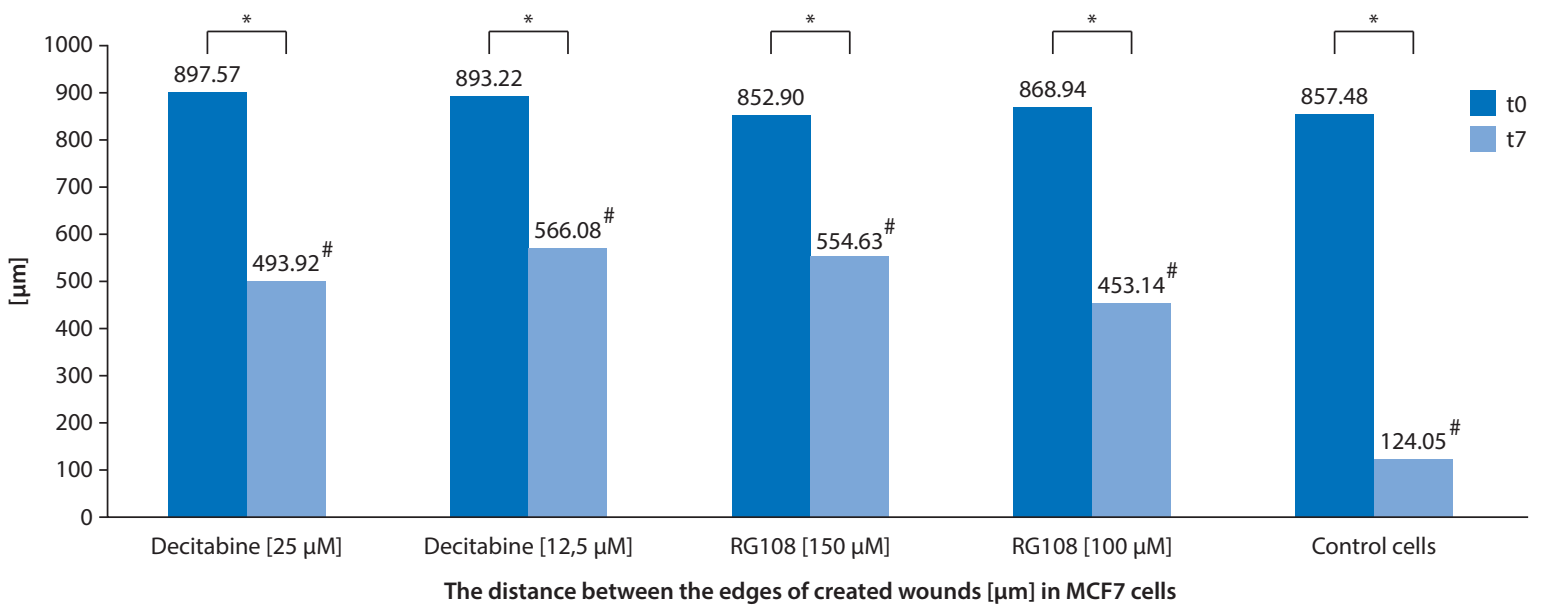

Figure 6. Changes in the average distance between the edges of created wounds in MCF7 cells [um] treated with DNMTi and in control cells, *, \# statistically significant results $(p<0.001)$

MCF7 cells after 7 days compared to time zero and compared to control cells were statistically significant.

\section{DISCUSSION}

Since genetic alterations are almost impossible to reverse, the potential reversibility of epigenetic regulatory mechanisms makes them attractive candidates for new targets in the effort of prevention and treatment of ovarian and breast carcinoma. Aberrant DNA CpG island methylation within genes' promoter region represents one possible mechanism of gene silencing by cancer [20]. The promoter methylation is related to reduction of DIRAS3 expression in most ovarian cancer and breast cancer cells [8, 21]. Ovarian and breast cancer cells treated with DNA demethylating agents can reactivate both the silenced and the imprinted alleles of DIRAS3. DIRAS3 is a cell cycle regulator that inhibits cancer cell growth, decreases motility and regulates tumor dormancy, all of which may indirectly depend on interaction with a transcription factor STAT3 with a potential oncogenic role. STAT3 is frequently phosphorylated and activated in the majority of breast and ovarian cancers $[22,23]$. Barrow et al. have observed increasing variation in DNA methylation across breast tissue starting from normal through benign to cancer tissue ending with even more aberrant methylation of DIRAS3 in 36\% patients with invasive breast cancer [24]. Lu et al. have indicated that loss of DIRAS3 expression in ovarian and breast cancer cells might be due to several mechanisms eg., maternal imprinting, loss of heterozygosity, promoter DNA methylation or decreased histone $\mathrm{H} 3$ acetylation [25].

Nishimoto et al. have indicated that activated STAT3 has been observed in most invasive breast cancers and in many breast and ovarian cancer cell lines but not in normal cells [13]. High expression of STAT3 has been observed in many different ovarian cancer cell lines. Han et al. have observed that total STAT3 protein level is expressed, among 
others, in A2780 and SKOV-3 cells. However, the level of phosphorylated STAT3 (pSTAT3) was higher in SKOV-3 cells than in the A2780 cells [26]. Nishimoto et al. have also observed that DIRAS3 expression is decreased in breast and ovarian cancer cells and properly expressed in normal breast and ovarian cells. At the same time they have indicated that DIRAS3 associates with STAT3 and plays an important role in down-regulating STAT3 activity [13]. Our study confirmed that DIRAS3 expression was in fact reduced in ovarian and breast cancer cell lines while STAT3 was highly expressed. Therefore, decreasing of DIRAS3 expression in ovarian and breast cancer cells may provide the mechanism that contributes to high constitutive STAT3 activity [13]. In a study comparing the anti-proliferative activity of two demethylating agents - 5-azacitidine (AZA) and 5-aza-2'-deoxycytidine (decitabine, DAC) and two HDAC inhibitors - suberoylanilide hydroxamic acid (SAHA) and trichostatin A (TSA) Chen et al. report that after 5 days of treatment with DAC the reduction in viability at increasing doses could be fitted by hyperbolic curves which decreased to asymptotes at approximately $30 \%$ for Hey and $60 \%$ for SKOv3 cells. There was no further increase in DAC's anti-proliferative activity at concentrations higher than $32 \mu \mathrm{M}$ in the SKOV-3 cells [27].

In turn, Feng et al. have observed an 8-fold increase of DIRAS3 expression in OVCA420 ovarian cancer cells treated with 5-aza-dC compared to approximately 2- to 4-fold increase in Hey, SKOV-3 or OVCA432 cells. Interestingly, DIRAS3 expression was not affected in CAOv3 cells when treated with demethylating agents [28].

In this study we have observed a statistically significant dose dependent effect of decitabine and RG108 on DIRAS3 expression in A2780 cells. Interestingly, lower doses of decitabine have activated DIRAS3 expression slightly more compared to control cells. This is supported by Mund et al. and Gil et al. who indicate that decitabine in lower doses might induce demethylation even more effectively with lower cytotoxicity [29, 30]. RG108 in turn has activated expression of DIRAS3 proportionally on the dose dependent manner. Yet, there was no increase of the DIRAS3 expression in MCF7 cells treated with decitabine or RG108. We observed no changes in the STAT3 expression after decitabine $(12.5 \mu \mathrm{M})$ and RG108 treatment when compared to A2780 control cells. What is more, compared to control cells decitabine at the dose $25 \mu \mathrm{M}$ has decreased the expression of STAT3 in A2780 cells. Similarly, both DNMTi treatment showed no influence on the activation of STAT3 expression in MCF7 cells.

In this study have also observed a dose dependent influence of decitabine and RG108 on the in vitro migration of A2780 and MCF7 cells. Both DNMTi have significantly ( $p<0.001$ ) reduced the speed of A2780 and MCF7 cell migration in wound healing assay in comparison to un- treated control cells cultured under standard conditions after 7 days. However, A2780 cells treated with decitabine [ $25 \mu \mathrm{M}$ and $12.5 \mu \mathrm{M}$ ] covered the wound respectively 3.5 and 2.5 times slower than MCF7 cells while A2780 cells treated with RG108 [150 $\mu \mathrm{M}$ and $100 \mu \mathrm{M}]$ covered the wound 6 and 3 times slower than MCF7 cells. A2780 and MCF7 control cells covered the wound with the same speed. To sum up, when the speed of wound healing is considered, human ovarian carcinoma cells show higher sensitivity to DNMTi compared to human breast cancer cells.

\section{Acknowledgment}

The study was supported by the funds: KNW-2-015/N/4/K, KNW-2-014/N/5/N, KNW-2-B10/N/6/K, KNW-2-B20/N/7/N, KNW-1-043/N/6/B.

\section{Conflict of interest}

The authors have no potential conflicts of interest to declare.

\section{REFERENCES}

1. Dai Z, Kosuri K, Otterson GA. Mechanisms of DNA demethylating drugs against cancer progression. In: Esteller M. ed. DNA Methylation, Epigenetics and Metastasis. Springer, United States 2005: 244-267.

2. Subramaniam D, Thombre R, Dhar A, et al. DNA methyltransferases: a novel target for prevention and therapy. Front Oncol. 2014; 4: 80, doi: 10.3389/fonc.2014.00080, indexed in Pubmed: 24822169.

3. Earp MA, Cunningham JM. DNA methylation changes in epithelial ovarian cancer histotypes. Genomics. 2015; 106(6): 311-321, doi: 10.1016/j. ygeno.2015.09.001, indexed in Pubmed: 26363302.

4. Győrffy B, Bottai G, Fleischer T, et al. Aberrant DNA methylation impacts gene expression and prognosis in breast cancer subtypes. Int J Cancer. 2016; 138(1):87-97, doi: 10.1002/ijc.29684, indexed in Pubmed: 26174627.

5. Esteller M. Epigenetic gene silencing in cancer: the DNA hypermethylome. Hum Mol Genet. 2007; 16 Spec No 1: R50-R59, doi: 10.1093/hmg/ddm018, indexed in Pubmed: 17613547.

6. Luo RZ, Peng H, Xu F, et al. Genomic structure and promoter characterization of an imprinted tumor suppressor gene ARHI. Biochim Biophys Acta. 2001; 1519(3): 216-222, doi: 10.1016/s0167-4781(01)00226-3, indexed in Pubmed: 11418188.

7. Rosen DG, Wang L, Jain AN, et al. Expression of the tumor suppressor gene ARHI in epithelial ovarian cancer is associated with increased expression of p21WAF1/CIP1 and prolonged progression-free survival. Clin Cancer Res. 2004; 10(19): 6559-6566, doi: 10.1158/1078-0432.CCR04-0698, indexed in Pubmed: 15475444

8. Li J, Cui G, Sun Lu, et al. STAT3 acetylation-induced promoter methylation is associated with downregulation of the ARHI tumor-suppressor gene in ovarian cancer. Oncol Rep. 2013; 30(1): 165-170, doi: 10.3892/or.2013.2414, indexed in Pubmed: 23604529.

9. Lu Z, Bast RC. The tumor suppressor gene ARHI (DIRAS3) inhibits ovarian cancer cell migration through multiple mechanisms. Cell Adh Migr. 2013; 7(2): 232-236, doi: 10.4161/cam.23648, indexed in Pubmed: 23357870.

10. http://www.uniprot.org/uniprot/O95661\#expression.

11. Yang J, Chatterjee-Kishore M, Staugaitis SM, et al. Novel roles of unphosphorylated STAT3 in oncogenesis and transcriptional regulation. Cancer Res. 2005; 65(3): 939-947, indexed in Pubmed: 15705894.

12. Muthu K, Panneerselvam M, Topno NS, et al. Structural perspective of ARHI mediated inhibition of STAT3 signaling: an insight into the inactive to active transition of ARHI and its interaction with STAT3 and importin $\beta$. Cell Signal. 2015; 27(4): 739-755, doi: 10.1016/j.cellsig.2014.11.036, indexed in Pubmed: 25499977.

13. Nishimoto A, YuY, Lu Z, et al. A Ras homologue member I directly inhibits signal transducers and activators of transcription 3 translocation and activity in human breast and ovarian cancer cells. Cancer Res. 2005; 65(15): 6701-6710, doi: 10.1158/0008-5472.CAN-05-0130, indexed in Pubmed: 16061651

14. http://www.onkologia-online.pl/medicine. 
15. Gros C, Fahy J, Halby L, et al. DNA methylation inhibitors in cancer: recent and future approaches. Biochimie. 2012; 94(11): 2280-2296, doi: 10.1016/j.biochi.2012.07.025, indexed in Pubmed: 22967704.

16. Fahy J, Jeltsch A, Arimondo PB. DNA methyltransferase inhibitors in cancer: a chemical and therapeutic patent overview and selected clinical studies. Expert Opin Ther Pat. 2012; 22(12): 1427-1442, doi: 10.1517/13543776.2012.729579, indexed in Pubmed: 23033952.

17. Gnyszka A, Jastrzebski Z, Flis S. DNA methyltransferase inhibitors and their emerging role in epigenetic therapy of cancer. Anticancer Res. 2013; 33(8): 2989-2996, indexed in Pubmed: 23898051.

18. Heerboth S, Lapinska K, Snyder N, et al. Use of epigenetic drugs in disease: an overview. Genet Epigenet. 2014; 6: 9-19, doi: 10.4137/GEG. S12270, indexed in Pubmed: 25512710.

19. Livak KJ, Schmittgen TD. Analysis of relative gene expression data using real-time quantitative PCR and the 2(-Delta Delta $C(T)$ ) Method. Methods. 2001; 25(4): 402-408, doi: 10.1006/meth.2001.1262, indexed in Pubmed: 11846609.

20. Bai H, Cao D, Yang J, et al. Genetic and epigenetic heterogeneity of epithelial ovarian cancer and the clinical implications for molecular targeted therapy. J Cell Mol Med. 2016; 20(4): 581-593, doi: 10.1111/jcmm.12771, indexed in Pubmed: 26800494.

21. LiY, Shi Li, Han C, et al. Effects of ARHI on cell cycle progression and apoptosis levels of breast cancer cells. Tumour Biol. 2012; 33(5): 1403-1410, doi: 10.1007/s13277-012-0388-1, indexed in Pubmed: 22528939.

22. Yu Y, Fujii S, Yuan J, et al. Epigenetic regulation of ARHI in breast and ovarian cancer cells. Ann N Y Acad Sci. 2003; 983: 268-277, doi: 10.1111/j.1749-6632.2003.tb05981.x, indexed in Pubmed: 12724231.

23. Huang S, Chang InS, Lin W, et al. ARHI (DIRAS3), an imprinted tumour suppressor gene, binds to importins and blocks nuclear import of cargo proteins. Biosci Rep. 2009; 30(3): 159-168, doi: 10.1042/BSR20090008, indexed in Pubmed: 19435463.

24. Barrow TM, Barault L, Ellsworth RE, et al. Aberrant methylation of imprinted genes is associated with negative hormone receptor status in invasive breast cancer. Int J Cancer. 2015; 137(3): 537-547, doi: 10.1002/ijc.29419, indexed in Pubmed: 25560175.

25. Lu Z, Luo RZ, Peng $\mathrm{H}$, et al. Transcriptional and posttranscriptional down-regulation of the imprinted tumor suppressor gene ARHI (DRAS3) in ovarian cancer. Clin Cancer Res. 2006; 12(8): 2404-2413, doi: 10.1158/1078-0432.CCR-05-1036, indexed in Pubmed: 16638845.

26. Han Z, Feng J, Hong Z, et al. Silencing of the STAT3 signaling pathway reverses the inherent and induced chemoresistance of human ovarian cancer cells. Biochem Biophys Res Commun. 2013; 435(2): 188-194, doi: 10.1016/j.bbrc.2013.04.087, indexed in Pubmed: 23665025.

27. Chen MY, Liao WSL, Lu Z, et al. Decitabine and suberoylanilide hydroxamic acid (SAHA) inhibit growth of ovarian cancer cell lines and xenografts while inducing expression of imprinted tumor suppressor genes, apoptosis, G2/M arrest, and autophagy. Cancer. 2011; 117(19): 4424-4438, doi: 10.1002/cncr.26073, indexed in Pubmed: 21491416.

28. Feng W, Marquez RT, Lu Z, et al. Imprinted tumor suppressor genes ARHI and PEG3 are the most frequently down-regulated in human ovarian cancers by loss of heterozygosity and promoter methylation. Cancer. 2008; 112(7): 1489-1502, doi: 10.1002/cncr.23323, indexed in Pubmed: 18286529.

29. Mund C, Brueckner B, Lyko F. Reactivation of epigenetically silenced genes by DNA methyltransferase inhibitors: basic concepts and clinical applications. Epigenetics. 2006; 1(1): 7-13, doi: 10.4161/epi.1.1.2375, indexed in Pubmed: 17998812.

30. Gil L, Mądry K, Komarnicki M. Mechanizmy działania leków hipometylujacych wzespołach mielodysplastycznych. Hematologia. 2012;3(2): 120-126. 